\title{
Fragilité-comorbidité : une association déterminante de la prise en charge d'une pathologie chez le sujet âgé
}

\author{
Frailty-comorbidity: a key association of pathology support in the elderly
}

\author{
P. Chassagne \\ C) Springer-Verlag France 2012
}

Parmi les caractéristiques retenues précisant la typologie des malades relevant d'une filière gériatrique on retrouve les notions : d'âge (supérieur à 75 ans), de polypathologie, de risque élevé de dépendance et/ou d'autonomie, ou encore la prévalence de syndromes gériatriques. On doit y ajouter désormais, dans la mesure où cette entité est depuis les années 1980 bien identifiée, l'existence d'une fragilité.

La fragilité est un état dynamique secondaire schématiquement à un épuisement progressif des réserves d'un individu. La fragilité qui affecte près de $20 \%$ des sujets après 80 ans n'est pas synonyme de comorbidité, elle est distincte de la dépendance et enfin n'est pas nécessairement irréversible.

Si la fragilité ne doit pas être confondue avec la comorbidité ces deux conditions sont intimement liées. On peut même parler d'une interaction entre ces deux éléments. En premier lieu, une fragilité s'accompagne par définition d'un risque accru de décompensations fonctionnelles en cascade. Ainsi une maladie infectieuse telle qu'une pneumopathie surviendra avec prédilection chez des sujets âgés dont l'état immunitaire est altéré autrement dit à ce niveau « fragile». De même, la survenue de chutes parfois compliquées de fracture dont la redoutable fracture de l'extrémité supérieure du fémur (FESF) est aussi le témoin d'une fragilité latente. On peut alors relier ces deux éléments. Une malnutrition protéino-énergétique, pierre angulaire du concept de fragilité tel qu'il a été défini par L. Fried, expose à une amyotrophie, s'accompagne de carence en micronutriment (exemple : vitamine D) dont la carence favorise la fragilité osseuse (synonyme d'ostéoporose) et contribue immanquablement à la survenue précoce de fracture. Si l'on considère la survenue d'un état confusionnel, dont la prévalence en soins gériatriques est majeure, on sait maintenant qu'il s'agit d'un témoin de fragilité cognitive sous jacente.

En second lieu, cette interaction fragilité-comorbidité doit être considérée quant à son impact négatif potentiel sur la récupération fonctionnelle et le reconditionnement physique des sujets âgés fragiles après la survenue d'un stress, autrement dit d'une pathologie aiguë. Un stress, qu'il soit infectieux, cognitif, chirurgical ou encore d'ordre métabolique en rapport avec une malnutrition de cinétique rapide liée par exemple à un état d'hypercatabolisme, est une circonstance classique de révélation d'un état de fragilité volontiers méconnu faute d'un dépistage. La traduction de cet état de fragilité latente s'observera lors de la phase de reconditionnement. Elle sera grevée de décompensations en cascade, source d'une morbidité-mortalité élevée. Négliger cette valence de fragilité c'est immanquablement s'exposer à une guérison plus difficile, incomplète et prolongée. En d'autres termes, c'est l'ensemble du reconditionnement physique et/ou fonctionnel du sujet âgé malade et par ailleurs fragile qui deviendra alors problématique.

Dans ce numéro des Cahiers de l'année gérontologique, nous avons souhaité illustrer autour de quatre situations, nutrition, FESF, infectiologie et cognition, cette intime relation entre comorbidité et fragilité. Un des messages que nous souhaitons de nouveau délivrer est celui de l'impérative nécessité de détecter précocement les caractéristiques de fragilité d'une personne âgée indissociables du projet de soins spécifique qu'elle requiert. Cette pratique doit être promue dans les filières gériatriques et bien entendu dans les unités de soins de spécialité non gériatriques.
P. Chassagne $(\bowtie)$

Service de médecine interne gériatrique, CHU de Rouen, 1, rue de Germont, F-76031 Rouen cedex, France

e-mail : philippe.chassagne@chu-rouen.fr 